\title{
Review of Vinflunine Data and Ongoing Developments in Urothelial Carcinoma
}

\author{
Anders Ullén \\ Department of Oncology-Pathology, Karolinska Institute, Stockholm, Sweden
}

\section{Overview of Registration Trials Data in Urothelial Carcinoma}

In total, 450 patients were treated in two phase II trials and one phase III trial that together formed the basis for the registration of vinflunine monotherapy in urothelial carcinoma patients after failure of platinumbased therapy (see Table 1). ${ }^{1-3}$

Similar data were reported in the two phase $\|$ trials, in terms of overall response rate (ORR, 5-18\%), median overall survival (OS, 6.6-8.2 months) and median progression-free survival (PFS, 2.83.0 months). ${ }^{2,3}$

In the core phase III study, results from the first analysis were reported following 1.8 years of follow-up. ${ }^{1}$ The objective of achieving a 2-month survival advantage was achieved. Os in the vinflunine plus best supportive care (BSC) arm was 6.9 months (95\% confidence interval [Cl] 5.7-8.0) versus 4.6 months (95 \% Cl 4.1-7.0) in the BSC-only arm, although the data were not statistically significant $(p=0.2868)$ in the intent-to-treat (ITT) population. However, after adjusting for prespecified prognostic factors that were outlined in the protocol (alkaline phosphatase and haemoglobin [Hb] levels, visceral involvement, performance status [PS], presence of lymph node [LN] metastases and pelvic radiotherapy), the difference in os between the two arms reached statistical significance in the eligible patient population, defined by the exclusion of 13 patients with at least one major protocol violation at baseline. A statistically significant $(p=0.04)$ 2.6 month difference in median OS was observed in the vinflunine arm: 6.9 months (95\% Cl 5.7-8.0) versus 4.3 months in the BSC arm (95\% Cl 3.8-5.4) (see Table 2).

A multivariate (ITT) analysis adjusting for prognostic factors, such as PS or alkaline phosphatase, confirmed the clearly statistically significant ( $p=0.036)$ impact of vinflunine treatment on OS, with a $23 \%$ reduction in the risk of death compared with the BSC-only arm (hazard ratio $[\mathrm{HR}]=0.77,95 \% \mathrm{Cl} 0.61-0.98)$ (see Table 2). ${ }^{1}$

An updated survival analysis of over 3.5 years' median follow-up in the ITT population was presented at the European Association of Urology (EAU) 2010 conference. ${ }^{4}$ The median survival advantage of 2.3 months was confirmed in the vinflunine plus BSC arm, although the difference was still not significant. In the eligible population, a statistically significant ( $p=0.0227$ ) difference of 2.6 months in median survival was observed in the vinflunine arm, and risk of death was reduced by $22 \%$. Thus, the 2-month difference in OS was maintained for the ITT and eligible populations (statistically significant in the eligible population): ${ }^{4}$ of note, it was possible to observe patients in the vinflunine arm surviving over 40 months. Even though they were only a small fraction of the total cohort, it may be interesting to characterise these patients in more detail.

In terms of secondary endpoints, all results favoured the vinflunine arm. Significant and meaningful benefits were observed in all main efficacy parameters, including ORR, PFS and disease-control rate (DCR) (see Table 3). ${ }^{4}$ Median PFS was doubled in the vinflunine arm compared with the BSC arm, and although the times are relatively short (3.0 versus 1.5 months, respectively), this improvement might be a clinically meaningful benefit in this patient population.

Furthermore, patient quality of life (QoL) was not impaired by vinflunine. Global health status measurements (European Organisation for Research and Treatment of Cancer [EORTC] QLQ-C30) showed a tendency towards better QoL for vinflunine plus BSC compared with BSC alone, although differences between the two treatment arms were not significant $(p=0.658)$. Importantly, a statistically significant evolution of the pain scale between the two treatment arms favouring the vinflunine arm was measured by week 12 of therapy $(p=0.046)$. In addition, less palliative radiotherapy was used in the vinflunine arm compared with BSC alone (4 \% versus $24 \%$ ). ${ }^{4}$

Grade 3 and grade 4 adverse events (AES) following vinflunine treatment in non-transitional cell carcinoma of the urothelial tract (non-TCCU) versus TCCU patients showed that myelosuppression was common and higher in TCCU patients than in non-TCCU patients. Non-haematological toxicity mainly included asthaenia/fatigue and constipation, and was also higher in TCCU patients compared with non-TCCU patients. Reasons for these differences between cancer patients may be related to age, previous treatment, aggressiveness of the disease or a combination of these factors (see Table 4). Overall, vinflunine has a good tolerability profile that is consistent with expected class effects.

\section{Additional Data Derived from the Pivotal Phase III Vinflunine Trial}

The pivotal phase III study produced interesting data demonstrating that the main adverse prognostic factors for OS in patients who have failed a platinum-based regimen were $\mathrm{Hb}<10 \mathrm{~g} / \mathrm{dl}$, the presence of liver metastases and PS $>0 .{ }^{5}$ Patients harbouring a combination of all three risk factors had a worse OS compared with those who had none (see Figure 1).

These data are important because they shed some light on which patients would most benefit from vinflunine therapy. These results were corroborated by Niegisch et al., ${ }^{6}$ who confirmed that accumulation of these adverse prognostic factors resulted in worse OS. This study also 
Table 1: Overview of Trials that Led to Vinflunine Registration ${ }^{1-3}$

\begin{tabular}{|c|c|c|c|c|}
\hline Trial & Design & Number of Treated Patients & Initial Dose (mg/m²; q3w) & Efficacy Endpoints \\
\hline Culine et al. ${ }^{1}$ & $\begin{array}{l}\text { Phase II, open-label, } \\
\text { single-arm VFL as second line }\end{array}$ & 51 & 320 & $\begin{array}{l}\text { Primary: ORR } \\
\text { Secondary: duration of response, PFS, OS }\end{array}$ \\
\hline Vaughn et al. ${ }^{2}$ & $\begin{array}{l}\text { Phase II, open-label, single-arm } \\
\text { VFL as second line }\end{array}$ & 151 & 320 or 280 & $\begin{array}{l}\text { Primary: ORR } \\
\text { Secondary: duration of response, } \\
\text { time to response, DCR, PFS, OS }\end{array}$ \\
\hline Bellmunt et al. ${ }^{3}$ & $\begin{array}{l}\text { Phase III, open-label, } \\
\text { randomised VFL as second line } \\
\text { VFL+BSC } \\
\text { BSC alone }\end{array}$ & $\begin{array}{l}248 \\
117\end{array}$ & 320 or 280 & $\begin{array}{l}\text { Primary: OS } \\
\text { Secondary: patient and clinical benefit, ORR, } \\
\text { time to response, duration of response, } \\
\text { DCR, PFS }\end{array}$ \\
\hline
\end{tabular}

highlighted lymph node (LN) disease as a prognostic factor. Of note, the population also included patients who had only received neo-adjuvant or adjuvant therapy. The validation of these prognostic risk factors is important because many physicians assume that second-line patients should not be treated, whereas patients with no or one adverse risk factor clearly benefit from second-line treatment. Conversely, secondline treatment appears to be not beneficial in patients who have all three risk factors. Naturally, these stratification factors are useful for conducting clinical trials, but are not predictive of treatment efficacy in clinical practice.

Further information derived from the 357 eligible patients enrolled in the pivotal phase III study evaluated whether prior platinum-based therapy in the first-line setting impacted on the subsequent benefit conferred by vinflunine treatment. In the phase III trial, $70.3 \%$ of patients received cisplatin, and $29.7 \%$ were pre-treated with another platinum-based regimen (carboplatin or oxaliplatin). Multivariate analysis showed that the nature of prior platinum-based therapy had no impact, but that the known prognostic factors (liver involvement, Hb level and PS) may explain differences in survival outcome in patients undergoing secondline therapy.

Moreover, Krajewski and co-workers studied the optimal tumour size variation threshold to predict os for patients receiving secondline vinflunin. ${ }^{8}$ Analysis of the 179 eligible patients treated with vinflunine who had at least one target lesion measurement in the phase III study showed that patients with sum long axis diameter (SLD) reduction of $\geq 10 \%$ had a significantly longer OS than those with SLD reduction of $<10 \%$ (11.3 versus 6.9 months; $p=0.0224)$. Thus, a minor reduction ( $\geq 10 \%$ tumour shrinkage) in SLD at first follow-up imaging was a better early predictor of outcome than the response evaluation criteria in solid tumours (RECIST version 1.0). ${ }^{8}$ These data are in line with clinical experience, as patients who respond with disease stabilisation or better are those in whom continued vinflunine treatment is considered, provided that it is tolerated. Therefore, another 'response' threshold may be more useful than RECIST in clinical routine practice.

\section{Vinflunine Use in Special Patient Populations}

Special patient populations include those with renal or hepatic impairment, and elderly patients. These conditions are not contraindications to vinflunine use, but require dose adjustments. In the case of renal impairment, the dose needs adjusting according to creatinine clearance $(\mathrm{CrCl})$ values. In patients whose $\mathrm{CrCl}$ is $\geq 60 \mathrm{ml} /$ minute, standard vinflunine dosing of $320 \mathrm{mg} / \mathrm{m}^{2}$ every 3 weeks (q3w) is recommended, or $280 \mathrm{mg} / \mathrm{m}^{2}$ q3w in patients who are PS 1 or who have received prior radiotherapy. A similar lower dose of $280 \mathrm{mg} / \mathrm{m}^{2}$
Table 2: Median Overall Survival after 1.8 Years of Follow-up ${ }^{1}$

\begin{tabular}{llll} 
& VFL+BSC & $p$ & BSC \\
Randomised Patients (Number) & $\mathrm{n}=253$ & & $\mathrm{n}=117$ \\
\hline Median follow-up (months) & 21.5 & & 22.3 \\
\hline Median OS (ITT) (months) & 6.9 & $\mathrm{p}=0.2868$ & 4.6 \\
[95\% CI] & {$[5.7-8.0]$} & & {$[4.1-7.0]$} \\
Median OS (eligible) (months) & 6.9 & $\mathrm{p}=0.0403$ & 4.3 \\
[95\% Cl] & {$[5.7-8.0]$} & & {$[3.8-5.4]$} \\
\hline Treatment effect on OS & HR=0.77 & $\mathrm{p}=0.036$ & \\
(multivariate analysis, ITT) & {$[0.61-0.98]$} & & \\
[95\% Cl] & Risk reduction: $23 \%$ &
\end{tabular}

$B S C=$ best supportive care; $\mathrm{Cl}=$ confidence interval; $H R=$ hazard ratio; $I T T=$ intent-totreat; $O S=$ overall survival; $V F L=$ vinflunine.

\section{Table 3: Secondary Endpoints ${ }^{4}$}

\begin{tabular}{llll} 
& VFL+BSC & $p$ & BSC \\
Randomised Patients (Number) & $\mathrm{n}=253$ & & $\mathrm{n}=117$ \\
\hline ORR (\%) & 8.6 & $\mathrm{p}=0.0063$ & 0 \\
{$[95 \% \mathrm{Cl}]$} & {$[5.0-13.7]$} & & \\
\hline $\mathrm{DC}(\%)$ & 41.1 & $\mathrm{p}=0.0024$ & 24.8 \\
{$[95 \% \mathrm{Cl}]$} & {$[35.0-47.4]$} & & {$[17.3-33.6]$} \\
\hline Median duration of & 7.4 & & \\
response (months) & & & \\
{$[95 \% \mathrm{Cl}]$} & {$[4.5-17.0]$} & & 4.2 \\
Median duration of DC (months) & 5.7 & & {$[3.8-4.9]$} \\
{$[95 \% \mathrm{Cl}]$} & {$[5.0-6.3]$} & & 1.5 \\
\hline Median PFS (months) & 3.0 & $\mathrm{p}=0.0012$ & {$[1.4-2.3]$} \\
{$[95 \% \mathrm{Cl}]$} & {$[2.1-4.0]$} & &
\end{tabular}

$\mathrm{Cl}=$ confidence interval; $\mathrm{DC}=$ disease control; $O R R=$ overall response rate; $P F S=$ progression-free survival.

q3W is recommended in patients whose $\mathrm{CrCl}$ is $40-60 \mathrm{ml} / \mathrm{minute}$, and a further reduced dose of $250 \mathrm{mg} / \mathrm{m}^{2} \mathrm{q} 3 \mathrm{w}$ in patients whose $\mathrm{CrCl}$ is 20-40 $\mathrm{ml} /$ minute. Vinflunine can be used in patients with hepatic impairment, provided it is not severe. Doses of $250 \mathrm{mg} / \mathrm{m}^{2}$ or $200 \mathrm{mg} /$ $\mathrm{m}^{2} \mathrm{q} 3 \mathrm{w}$ are recommended in patients with mild Child-Pugh Grade A and moderate (Grade B) impairment, respectively.

Vinflunine should be administered at $280 \mathrm{mg} / \mathrm{m}^{2} \mathrm{q} 3 \mathrm{w}$ in patients who are 75-79 years old, and the dose should be lowered to $250 \mathrm{mg} / \mathrm{m}^{2}$ q3w in patients $\geq 80$ years old who are in good shape. Of note, these are recommendations, and the decision on which dose to administer depends on biological parameters. Upper age thresholds are currently not outlined in clinical trials. In addition, it may be of value to collect safety data in patients with low $\mathrm{CrCl}$ who have received vinflunine, as there is a possibility that the $\mathrm{CrCl}$ thresholds for vinflunine dose adjustments may be lowered. 


\section{Table 4: Tolerability Profile (Summary of Product Characteristics)}

\begin{tabular}{lll} 
Related AEs Grade 3/4 (\% Patients) & & \\
& Non-TCCU & TCCU \\
Haematological events & 743 patients & 445 patients \\
\hline Neutropenia & 47.6 & 54.6 \\
\hline Leucopenia & 30.8 & 45.2 \\
\hline Anaemia & 6.3 & 17.3 \\
\hline Febrile Neutropenia & 4.4 & 6.7 \\
\hline Non-haematological events & 753 patients & 450 patients \\
Asthenia/fatigue & 12.1 & 15.8 \\
\hline Constipation & 10.0 & 15.3 \\
\hline Abdominal pain & 6.3 & 4.9 \\
\hline Neutropenic infection & 1.5 & 4.0 \\
\hline Myalgia & 3.5 & 3.1 \\
\hline Vomiting & 2.9 & 2.9 \\
\hline Nausea & 2.9 & 2.9 \\
\hline Anorexia & 1.9 & 2.7 \\
\hline Injection site reaction & 0.4 & 0.4
\end{tabular}

$A E=$ adverse events; $T C C U=$ transitional cell carcinoma of the urothelium

Figure 1: Overall Survival Stratified by Risk Group (Zero, One, Two or Three Risk Factors) ${ }^{5}$

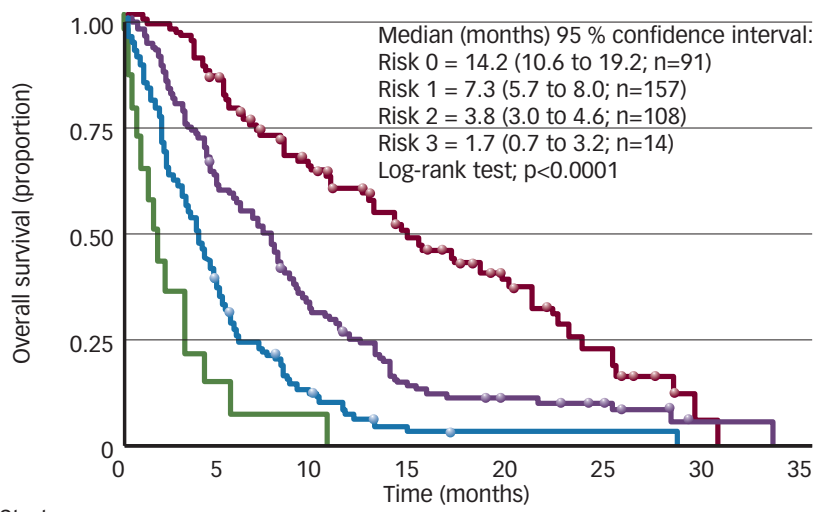

Strata

$\begin{array}{rll}\text { Risk }=0 & \text { Censored risk }=0 & \text { Risk }=1 \\ \text { Risk }=2 & \text { Risk }=3\end{array}$

\section{Vinflunine Clinical Profile in Advanced Urothelial Carcinoma - European Guidelines}

Vinflunine demonstrated a significant and clinically relevant survival advantage in the pivotal phase III trial, of 6.9 months versus 4.3 months in the BSC-only arm ( $p=0.0403)$. Consistent, robust and clinically relevant efficacy was shown in the phase II and phase III registration trials. Vinflunine also showed a predictable, acceptable and manageable tolerability profile, with no known cumulative toxicity. The benefit-risk ratio for vinflunine is positive overall. Moreover, prognostic factors for second-line treatment have been described and deserve consideration.

Due to vinflunine's favourable clinical profile, it is the recommended drug of choice in the guidelines of the EAU and of the European Society of Medical Oncology (ESMO) for second-line treatment of metastatic disease. In these guidelines, vinflunine is attributed the highest level of evidence and grade of recommendation for second-line use, although it is weakened by the matter of statistical significance in the ITT population, as outlined above..$^{9,10}$

The EAU guidelines recommend that vinflunine should be offered in patients progressing after platinum-based combination chemotherapy for metastatic disease. There is only one valid randomised phase III trial showing survival benefit in this setting. ${ }^{1.9}$ However, other more traditional second-line chemotherapies may also confer some benefit, even though activity of these regimens has not been strongly demonstrated in an evidence-based context with only short PFS durations. When considering monotherapy, vinflunine is an attractive choice, because it is the only approved regimen in Europe, and there are no randomised data for other drugs (e.g. docetaxel or paclitaxel), including patients who are eligible to receive second-line combination therapy (vinflunine combination regimens in the second-line setting are discussed below). The EAU guidelines add that alternative treatment within a clinical trial setting may also be offered..$^{10}$

In 'real-life' clinical practice, when deciding on a patient's treatment options, the assumption is that, even in the absence of data, younger and fitter patients may benefit from combination chemotherapy, and that these patients may be re-challenged with platinum-based chemotherapy. This explains why vinflunine is being used in some cases in the third-line setting in patients deemed eligible to receive platinum-based second-line therapy. As the EAU guidelines suggest, further trials are needed in the second-line setting, for instance, combining vinflunine with carboplatin, to see whether alternative regimens could confer survival benefit.

\section{Current Developmental Programme of Vinflunine in Urothelial Carcinoma}

In first-line chemotherapy, vinflunine is under evaluation in phase II studies in fit patients as single agent maintenance therapy (JAvlor switch in Maintenance [JASIMA] and Maintenance JAvlor [MAJA] trials), and as part of combination regimens in unfit patients (Javlor Association Study in TCCU [JASINT] and Northern Urology Cooperative Oncology Group 1 [NUCOG1] trials).

The JASINT trial randomised vinflunine and gemcitabine versus vinflunine and carboplatin, whereas the Nordic project NUCOG1 will randomise vinflunine plus gemcitabine versus gemcitabine and carboplatin in unfit patients. Patient recruitment is complete in JASINT.

The JASIMA and MAJA studies are two phase II maintenance trials following first-line chemotherapy that are currently recruiting. The JASIMA single arm trial will investigate vinflunine up to disease progression after first-line chemotherapy with up to four cycles of gemcitabine plus cisplatin (GC) treatment. Responding patients with stable disease or better will be included in the trial. Furthermore, the Spanish MAJA trial is a randomised study in which up to six cycles of primary treatment with GC were allowed. Use of vinflunine as maintenance therapy is a focus of interest. In the field of urology, this approach of switch maintenance is debated. A platinum-based chemotherapy without vinflunine, followed by vinflunine maintenance could be considered an early form of secondline treatment.

In patients who have received prior treatment with platinumbased chemotherapy (as first-line or after neoadjuvant or adjuvant chemotherapy), vinflunine is being tested in combination with targeted agents, or as part of non-platinum containing combinations. Three trials evaluating vinflunine are planned or ongoing in pre-treated patients. The NUCOG2 is a phase I trial that is designed to evaluate vinflunine plus pemetrexed, whereas the NUCOG3 phase I trial will test vinflunine plus a targeted agent (sorafenib). Finally, a planned vinflunine monotherapy trial - Javlor in Mexico (JAMEX) - will also test its efficacy in populations with ethnic specificities (in a Mexican population). 
Importantly, the NUCOG3 trial was designed with the objective of including a translational component. Tumour biopsies are being performed before the start of treatment and after one treatment cycle, and consecutive blood and urine samples are also being collected. It may be valuable, even in small exploratory trials, to collect samples that may help finetune patient selection that would help optimise treatment. Small trials may not result in striking differences between treatment arms, and data on biological markers would be strengthened, especially if a trend is observed.

\section{Conclusion}

Vinflunine, the first and only registered therapy for the management of advanced or metastatic TCCU after failure of a platinum-containing regimen in the first-line setting, increased the interest in clinical research on urothelial carcinoma. As such, vinflunine is recognised as the standard treatment in major European guidelines (EAU and ESMO) after failure of a platinum-based regimen.
Collecting data on vinflunine in combination regimens is a priority. Data from additional trials will help to better define and optimise the future role of vinflunine in TCCU. Data from ongoing and planned phase II trials in the first-line setting in both fit and unfit patients, and a series of trials in pre-treated patients are expected soon.

A number of other indications in which vinflunine could theoretically be evaluated have been proposed. These include vinflunine as a partner in perioperative treatment or primary first-line treatment, or in combination with other compounds to those currently tested in first-line fit patients and in the pre-treated patient population. Furthermore, it is becoming increasingly clear that evaluation methods could be improved, to allow responding patients to be discriminated as early as possible from nonresponders. Important challenges include a better understanding of the tumour biological basis for differences in responders and non-responders, and attempts to identify biomarkers. Together, these measures will help design better trials in the future and improve treatment.
1. Bellmunt J, Theodore C, Demkov T, Komyakov B, et al., Phase III trial of vinflunine plus best supportive care compared with best supportive care alone after a platinum-containing regimen in patients with advanced transitional cell carcinoma of the urothelial tract, I Clin Oncol, 2009;27(27):4454-61.

2. Culine S, Theodore C, De Santis M, Bui B, et al., A phase II study of vinflunine in bladder cancer patients progressing after first-line platinum-containing regimen, $\mathrm{Br} J$ Cancer, 2006;94(10):1395-401.

3. Vaughn DJ, Srinivas S, Stadler WM, Pili R, et al., Vinflunine in platinum-pretreated patients with locally advanced or metastatic urothelial carcinoma: results of a large phase 2 study, Cancer, 2009;115(18):4110-7.
4. Culine S, Lucas C, Salhi Y, Bellmunt J, Updated survival results of the phase III trial comparing vinflunine (V) to best supportiv care (BSC) in advanced transitional cell carcinoma of the care (BSC) in advanced transitional cell carcinoma of the
urothelium (TCCU) after failure of a prior platinum-containing regimen, Eur Urol Suppl, 2010;9(2):38.

5. Bellmunt J, Choueiri TK, Fougeray R, et al., Prognostic factors in patients with advanced transitional cell carcinoma of the urothelial tract experiencing treatment failure with platinumcontaining regimens, J Clin Oncol, 2010;28(11):1850-55.

6. Niegisch G, Fimmers R, Siener R, Park Sl, Albers P, Prognostic factors in second-line treatment of urothelial cancers with gemcitabine and paclitaxel (German Association of Urological Oncology trial AB20/99), Eur Urol, 2011;60(5):1087-96.
7. Fougeray R, Choueiri TK, Pons F, et al., Impact of first-line platinum therapy on survival in patients with platinumrefractory advanced transitional cell carcinoma of the urothelium (TCCU) treated with vinflunine, I Clin Oncol, 2012;30(15):e15007.

8. Krajewski KM, Fougeray R, Bellmunt J, Pons F, et al., Optimisation of the size variation threshold for imaging evaluation of response in patients with platinum-refractory advanced transitional cell carcinoma of the urothelium treated with vinflunine, Eur J Cancer, 2012;48(10):1495-502

9. Bellmunt J, Orsola A, Wiegel T, Guix M, et al., Bladder cancer: ESMO Clinical Practice Guidelines for diagnosis, treatment and follow-up, Ann Oncol, 2011;22(Suppl. 6):vi45-9. 